MS2 Development of new

types of sample preparation

(both XFEL \& synchrotrons)

Chairs: Jörg Standfuss, Gwyndaf Evans

\section{MS2-01 Enabling time-resolved structural studies of biological macromolecules}

Arwen Pearson ${ }^{1}$

\section{Hamburg Centre for Ultrafast Imaging, University of Hamburg \\ email: arwen.pearson@cfel.de}

In order to fully understand the mechanisms of biological processes, time-resolved methodologies that allow us to comprehend how function is linked to changes in molecular structure are required. Time-resolved X-ray crystallography provides a means of directly visualising structural rearrangements associated with function. Although time-resolved crystallography is a powerful tool it has not been widely applied to biomacromolecules, due to both the lack of beamlines where such experiments can be done as well as to the challenge of fast and uniform reaction initiation. To address these challenges we have developed a new multiplexing data collection method, based on the Hadamard transform, to make sub millisecond time-resolved data collection possible at standard macromolecular crystallography beamlines. We are also developing a suite of photochemical and rapid mixing tools for reaction initiation.

Keywords: time-resolved crystallography, rapid mixing, photocaging
MS2-O2 Structure determination of a membrane protein with data collected from micro-crystals in lipidic cubic phase at room temperature in low background CrystalDirectTM crystallization plates.

Thomas R. Schneider ${ }^{1}$, Gleb Bourenkov ${ }^{1}$, Maria Martinez-Molledo ${ }^{1}$, Ivars Karpics ${ }^{1}$, Esben M. Quistgaard ${ }^{2}$, Guillaume Hoffmann ${ }^{3}$, Florent Cipriani ${ }^{3}$, Josan Marquez ${ }^{3}$, Rob Meijers $^{1}$, Christian Loew ${ }^{1}$

1. European Molecular Biology Laboratory, Hamburg Outstation, c/o DESY, Notkestr. 85, 22603 Hamburg, Germany.

2. Laboratory Karolinska Institutet Biophysics/MBB Scheeles väg 2 SE - 17177 Stockholm, Sweden

3. European Molecular Biology Laboratory, Grenoble Outstation, 71 avenue des Martyrs, CS 90181, 38042 Grenoble Cedex 9, France.

email: thomas.schneider@embl-hamburg.de

Crystallization using the lipidic cubic phase (LCP) methodology in many cases is a decisive step towards obtaining high-resolution structural information for membrane proteins. However, harvesting crystals from LCP setups is notoriously difficult and frequently results in damaged or destroyed crystals. Furthermore, cryo-protection can be difficult, and data collection at cryogenic temperatures is often hampered by the LCP-matrix becoming opaque upon flash-cooling preventing optical crystal centering.

To overcome the above problems, we have grown crystals of a membrane transporter protein, PepT (491 residues; Lyons et al. 2014), in CrystalDirect ${ }^{\mathrm{TM}}$ plates and collected in situ diffraction data at room temperature on the EMBL beamline P14 at PETRA III (DESY, Hamburg). The $5 \mu \mathrm{m}$ micro-focus beam of $\mathrm{P} 14$ in combination with the low X-ray background of the CrystalDirect ${ }^{\mathrm{TM}}$ plates allowed to acquire diffraction data to $2.5 \AA$ resolution from crystals with typical dimensions of $10 \mu \mathrm{m}$ and embedded in LCP. Employing the serial helical scan data collection strategy (Gati, Bourenkov et al. 2014) as implemented in the high-precision MD3 diffractometer (ARINAX, Grenoble, France), 66 serial helical scans were performed on 19 crystallization wells delivering a total of 36013 frames containing more than 2 million reflections in 2 hours of beamtime.

The reflections were integrated, scaled, and merged into a $98 \%$ complete data set (space group $\mathrm{C} 222_{1}$ ) containing 23105 reflections to $2.5 \AA$. Structure solution by molecular replacement and refinement using standard methods delivered high-quality electron density maps and a high-quality model $\left(\mathrm{R}_{\text {work }} / \mathrm{R}_{\text {free }}=0.219 / 0.235\right)$ for the crystal structure of PepT ${ }_{\text {st }}^{\text {work }}$ at room temperature.

Gati, Bourenkov et al. (2014)Lyons et al. (2014) EMBO Rep. 15(8):886-93 


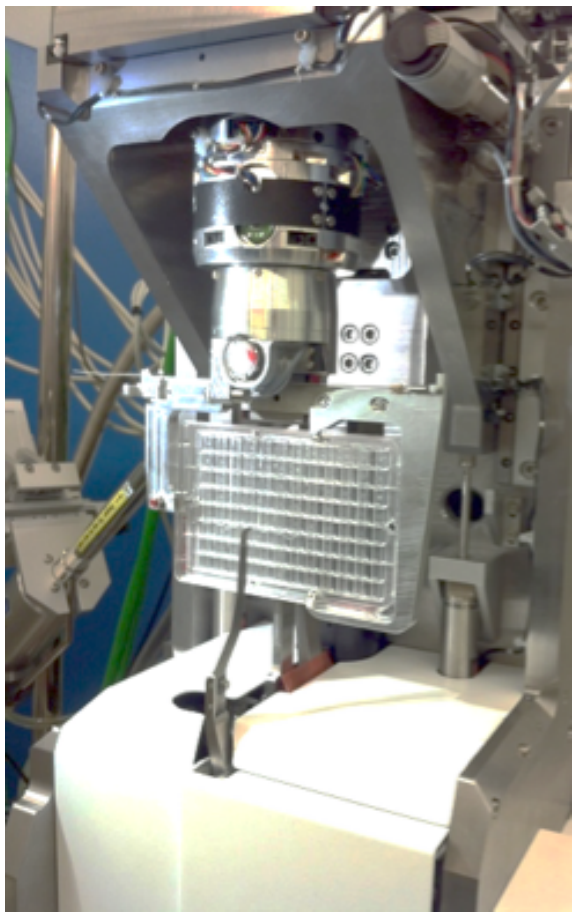

Figure 1. CrystalDirect ${ }^{\mathrm{TM}}$ crystallization plate mounted on the MD3 diffractometer for in situ data collection.

Keywords: in situ datacollection, LCP, serial crystallography

\section{MS2-O3 Lipidic Cubic Phase Injection for Serial X-ray Crystallography}

\author{
Daniel James ${ }^{1}$
}

1. Paul Scherrer Institut, Switzerland

email: daniel.james@psi.ch

High intensity $\mathrm{x}$-ray free electron laser (XFEL) pulses are destructive for biological samples. Serial femtosecond crystallography (SFX) experiments require sample to be replaced after each XFEL pulse, and have typically used liquid microjets to continuously renew sample. The high flow rate associated with these liquid microjets requires large volumes of sample, most of which runs to waste between shots. An injector designed to extrude a viscous gel-like material called lipidic cubic phase (LCP) was developed to address this problem. LCP, commonly used as a growth medium for membrane protein crystals, lends itself to low flow rate jetting and so reduces the amount of sample wasted significantly. Recently the injector has been used for serial crystallography experiments at synchrotrons, and has been shown to work with alternate viscous carrier fluids extending the usability of the injector.

Keywords: XFEL pulses, Lipidic Cubic Phase Injection 